\title{
POLYNOMIAL INTERPOLATION ON THE UNIT SPHERE II
}

\author{
WOLFGANG ZU CASTELL, NOEMÍ LAÍN FERNÁNDEZ AND YUAN XU
}

\begin{abstract}
The problem of interpolation at $(n+1)^{2}$ points on the unit sphere $\mathbb{S}^{2}$ by spherical polynomials of degree at $\operatorname{most} n$ is proved to have a unique solution for several sets of points. The points are located on a number of circles on the sphere with even number of points on each circle. The proof is based on a method of factorization of polynomials.
\end{abstract}

\section{INTRODUCTION}

Let $\mathbb{S}^{2}=\{x:\|x\|=1\}$ denote the unit sphere of $\mathbb{R}^{3}$, where $\|x\|^{2}=x_{1}^{2}+x_{2}^{2}+x_{3}^{2}$. Let $\Pi_{n}\left(\mathbb{S}^{2}\right)$ denote the space of spherical polynomials of degree $n$, which is the restriction of polynomials of degree $n$ in three variables to $\mathbb{S}^{2}$. It is known that

$$
\operatorname{dim} \Pi_{n}\left(\mathbb{S}^{2}\right)=(n+1)^{2}, \quad n \geq 0 .
$$

The problem of interpolation on the unit sphere by polynomials is as follows:

Problem 1. Let $X=\left\{\mathbf{a}_{i}: 1 \leq i \leq(n+1)^{2}\right\}$ be a set of pairwise distinct points on $\mathbb{S}^{2}$. Find conditions on $X$ such that there is a unique polynomial $T \in \Pi_{n}\left(\mathbb{S}^{2}\right)$ satisfying

$$
T\left(\mathbf{a}_{i}\right)=f_{i}, \quad \mathbf{a}_{i} \in X, \quad 1 \leq i \leq(n+1)^{2},
$$

where $\left\{f_{i}\right\}$ is an arbitrary set of data.

If there is a unique solution to the interpolation problem, we say that the problem is poised and that $X$ solves Problem 1 . This problem has been studied recently in [3, 4, 5, 7, 11, 14, 15].

Although almost all choices of $X$ will solve Problem 1, it is difficult to know whether a given set $X$ will work since computing the determinant of the interpolation matrix is difficult. In [14] a large family of sets of interpolation points is given explicitly, each set solving Problem 1. Let us briefly describe this construction. The $(n+1)^{2}$ points lie on $n+1$ distinct latitudes (parallel circles on $\mathbb{S}^{2}$ ), and each latitude contains an odd number of equidistant points. The number of points needs not to be the same on each latitude and there is no restriction on the position of the latitudes. For the simplest case $n=2 m$, the set of $(2 m+1)^{2}$ points lie on $2 m+1$ latitudes, each of them containing $2 m+1$ equally spaced nodes. In [4, another family of points that solves Problem 1 was found, for which $n=2 m-1$. There the points lie on $2 m$ latitudes and each latitude has an even number of $2 m$ equally spaced points. In this case, the $2 m$ latitudes are divided into two groups;

Date: August 18, 2018.

1991 Mathematics Subject Classification. 41A05, 41A63, 65D05.

Key words and phrases. Interpolation, spherical polynomials, unit sphere.

The second author was supported by the Graduate Program Applied Algorithmic Mathematics of the Munich University of Technology. The work of the third author was supported in part by the National Science Foundation under Grant DMS-0201669. 
the equidistant points on one half of the latitudes need to differ by a rotation from the points on the other half of the latitudes. While the proof in [4] is based on the analysis of the determinants of the interpolation matrix, the proof in [14] uses a factorization method which avoids the determinants. Furthermore, the factorization method provides many more sets of points leading to poised problems. A key observation in 14 is that the use of equidistant points allows us to reduce the problem on the sphere to a special trigonometric interpolation problem.

The purpose of this paper is to show that the factorization method also works in the setting of an even number of points on each latitude. Again, the use of equidistant points reduces the problem to an interpolation problem of one variable. However, the new interpolation problem is different from the one with an odd number of points on each latitude and has to be solved using a completely different method. In comparison to [4, the factorization method allows to obtain more sets of points that solve Problem 1.

For the background of polynomial interpolation in general, we refer to the survey article [6] and the references therein, even though interpolation on the sphere is not discussed there. Let us also mention that the factorization method is closely related to the method used for polynomial interpolation on the unit disk in 1, 2, 8. Apart from a result in [7, which is a simple consequence of Bezout's theorem, the family of points found in [4, 14, and those stated below appear to be the only ones that are given explicitly for all $n$.

The paper is organized as follows. The factorization method is studied in Section 2 and its application to polynomial interpolation on $\mathbb{S}^{2}$ is given in Section 3.

\section{FACTORIZATION OF POLYNOMIALS}

2.1. Polynomial representation. For fixed $a \in(-1,1)$, let $\mathbb{S}^{2}(a):=\{(x, y, z) \in$ $\left.\mathbb{S}^{2}: z=a\right\}$ denote the circle on $\mathbb{S}^{2}$ resulting from the intersection of $\mathbb{S}^{2}$ with the plane $z=a$. This set is called latitude at $z=a$.

On the unit sphere $\mathbb{S}^{2}$ it is more convenient to work with spherical coordinates,

$$
x=\sin \theta \sin \phi, \quad y=\sin \theta \cos \phi, \quad z=\cos \theta, \quad 0 \leq \phi<2 \pi, \quad 0 \leq \theta \leq \pi .
$$

For a polynomial $T_{n} \in \Pi_{n}\left(\mathbb{S}^{2}\right)$, we introduce the notation $\widetilde{T}_{n}$ defined by

$$
\widetilde{T}_{n}(\theta, \phi)=T_{n}(\sin \theta \cos \phi, \sin \theta \sin \phi, \cos \theta), \quad 0 \leq \phi<2 \pi, \quad 0 \leq \theta \leq \pi .
$$

If $X=\left\{\left(x_{i}, y_{i}, z_{i}\right): 1 \leq i \leq M\right\}$ is a set of points on $\mathbb{S}^{2}$, we also use the notation $\widetilde{X}=\left\{\left(\theta_{i}, \phi_{i}\right): 1 \leq i \leq M\right\}$ for the corresponding set of spherical coordinates.

It has been shown in Section 2 of 14 that the polynomial $\widetilde{T}_{n}$ can be written as

$$
\begin{aligned}
\widetilde{T}_{n}(\theta, \phi)= & a_{0}(\cos \theta) \\
& +\sum_{k=1}^{n}\left[a_{k}(\cos \theta)(\sin \theta)^{k} \cos k \phi+b_{k}(\cos \theta)(\sin \theta)^{k} \sin k \phi\right],
\end{aligned}
$$

where $a_{k}(\cdot)$ and $b_{k}(\cdot)$ are polynomials of degree $n-k$ in one variable. Note that for any fixed $\theta$, the polynomial $\widetilde{T}_{n}(\theta, \cdot)$ is a trigonometric polynomial of degree $n$.

Below we will consider interpolation problems based on points that are equidistantly distributed on an even number of latitudes, each of them containing an even number of nodes. To describe these points, it is convenient to introduce the 
following notation:

$$
\Theta_{\alpha, s}:=\left\{\phi_{j}^{\alpha}: \phi_{j}^{\alpha}=\frac{(2 j+\alpha) \pi}{2 s}, j=0,1, \ldots, 2 s-1\right\},
$$

where $s \in \mathbb{N}$ and $\alpha \in[0,2)$. These points can be considered as equidistant points on the unit circle using the mapping $\phi \mapsto e^{i \phi}$. The parameter $\alpha$ indicates that the points are defined up to a rotation by an angle of $\alpha \pi / 2 s$.

Lemma 2.1. Let $n=2 m-1$ and $\alpha \in[0,2)$. For $\phi \in \Theta_{\alpha, m}$,

$$
\begin{aligned}
& \widetilde{T}_{n}(\theta, \phi)=a_{0}(\cos \theta) \\
& +\sum_{k=1}^{m-1}\left[\left(a_{k}(\cos \theta)(\sin \theta)^{k}+u_{2 m-k}(\cos \theta)(\sin \theta)^{2 m-k}\right) \cos k \phi\right. \\
& \left.\quad+\left(b_{k}(\cos \theta)(\sin \theta)^{k}+v_{2 m-k}(\cos \theta)(\sin \theta)^{2 m-k}\right) \sin k \phi\right] \\
& +\left(a_{m}(\cos \theta) \cos \frac{\alpha \pi}{2}-b_{m}(\cos \theta) \sin \frac{\alpha \pi}{2}\right)(\sin \theta)^{m} \cos \left(m \phi-\frac{\alpha \pi}{2}\right),
\end{aligned}
$$

where, for $k=1, \ldots, m-1$,

$$
\begin{aligned}
& u_{2 m-k}(t)=a_{2 m-k}(t) \cos \alpha \pi+b_{2 m-k}(t) \sin \alpha \pi, \\
& v_{2 m-k}(t)=a_{2 m-k}(t) \sin \alpha \pi-b_{2 m-k}(t) \cos \alpha \pi
\end{aligned}
$$

are polynomials of degree $k-1$.

Proof. We split the sum in (2.1) into two sums, one over $1 \leq k \leq m-1$ and the other over $m \leq k \leq 2 m-1$. In the second sum we change the summation index $k \mapsto 2 m-k$ and use the elementary relations

$$
\begin{aligned}
\cos (2 m-k) \phi & =\cos ((2 j+\alpha) \pi-k \phi)=\cos \alpha \pi \cos k \phi+\sin \alpha \pi \sin k \phi, \\
\sin (2 m-k) \phi & =\sin ((2 j+\alpha) \pi-k \phi)=\sin \alpha \pi \cos k \phi-\cos \alpha \pi \sin k \phi,
\end{aligned}
$$

which holds for $\phi \in \Theta_{\alpha, m}$. Combining the two sums, we obtain

$$
\begin{array}{r}
\widetilde{T}_{n}(\theta, \phi)=a_{0}(\cos \theta)+\sum_{k=1}^{m-1}\left[\left(a_{k}(\cos \theta)(\sin \theta)^{k}+u_{2 m-k}(\cos \theta)(\sin \theta)^{2 m-k}\right) \cos k \phi\right. \\
\left.+\left(b_{k}(\cos \theta)(\sin \theta)^{k}+v_{2 m-k}(\cos \theta)(\sin \theta)^{2 m-k}\right) \sin k \phi\right] \\
+\left(a_{m}(\cos \theta) \cos (\alpha \pi-m \phi)+b_{m}(\cos \theta) \sin (\alpha \pi-m \phi)\right)(\sin \theta)^{m}
\end{array}
$$

Using the addition formula for the cosine and the sine function

$$
\begin{aligned}
& \cos (m \phi-\alpha \pi)=\cos \left(m \phi-\frac{\alpha \pi}{2}\right) \cos \frac{\alpha \pi}{2}+\sin \left(m \phi-\frac{\alpha \pi}{2}\right) \sin \frac{\alpha \pi}{2}, \\
& \sin (m \phi-\alpha \pi)=\sin \left(m \phi-\frac{\alpha \pi}{2}\right) \cos \frac{\alpha \pi}{2}-\cos \left(m \phi-\frac{\alpha \pi}{2}\right) \sin \frac{\alpha \pi}{2},
\end{aligned}
$$

the $a_{m}$ and $b_{m}$ terms of the above expression of $\widetilde{T}_{n}$ can be rewritten as

$$
\begin{aligned}
& \left(a_{m}(\cos \theta) \cos \frac{\alpha \pi}{2}-b_{m}(\cos \theta) \sin \frac{\alpha \pi}{2}\right)(\sin \theta)^{m} \cos \left(m \phi-\frac{\alpha \pi}{2}\right) \\
+ & \left(a_{m}(\cos \theta) \sin \frac{\alpha \pi}{2}+b_{m}(\cos \theta) \cos \frac{\alpha \pi}{2}\right)(\sin \theta)^{m} \sin \left(m \phi-\frac{\alpha \pi}{2}\right) .
\end{aligned}
$$

Consequently, formula (2.2) follows from the fact that $\phi \in \Theta_{\alpha, m}$ satisfies $\sin (m \phi-$ $\alpha \pi / 2)=0$. 
Lemma 2.2. Let $\widetilde{T}_{2 m-1}$ be given as in (2.2) and $\theta \in(0, \pi)$. If $\widetilde{T}_{2 m-1}(\theta, \phi)=0$ for $\phi \in \Theta_{\alpha, m}$, then $a_{0}(\cos \theta)=0$, and

$$
\begin{aligned}
& a_{k}(\cos \theta)+(\sin \theta)^{2 m-2 k}\left(a_{2 m-k}(\cos \theta) \cos \alpha \pi+b_{2 m-k}(\cos \theta) \sin \alpha \pi\right)=0, \\
& b_{k}(\cos \theta)+(\sin \theta)^{2 m-2 k}\left(a_{2 m-k}(\cos \theta) \sin \alpha \pi-b_{2 m-k}(\cos \theta) \cos \alpha \pi\right)=0,
\end{aligned}
$$

for $1 \leq k \leq m-1$. Furthermore,

$$
a_{m}(\cos \theta) \cos \frac{\alpha \pi}{2}-b_{m}(\cos \theta) \sin \frac{\alpha \pi}{2}=0 .
$$

Proof. The proof uses the following fact. Interpolation on the $2 m$ zeros of the

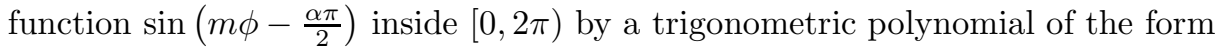

$$
S_{m-1}(\phi)=a_{0}+\sum_{k=1}^{m-1}\left(a_{k} \cos k \theta+b_{k} \sin k \theta\right)+a_{n} \cos (m \phi-\alpha \pi / 2)
$$

is unique (see [16, Vol. II]). Observe that the points in $\Theta_{\alpha, m}$ are exactly zeros of $\sin (m \phi-\alpha \pi / 2)$ and $\cos (m \phi-\alpha \pi / 2)= \pm 1$ for $\phi \in \Theta_{\alpha, m}$.

By Lemma2.1 $\widetilde{T}_{2 m-1}$ takes the form of (2.2). The assumption that $\widetilde{T}_{2 m-1}(\theta, \phi)=$ 0 implies that the coefficients of $\widetilde{T}_{2 m-1}(\theta, \cdot)$ are all zero. This leads to $a_{0}(\cos \theta)=0$,

$$
\begin{aligned}
& a_{k}(\cos \theta)(\sin \theta)^{k}+u_{2 m-k}(\cos \theta)(\sin \theta)^{2 m-k}=0, \\
& b_{k}(\cos \theta)(\sin \theta)^{k}+v_{2 m-k}(\cos \theta)(\sin \theta)^{2 m-k}=0,
\end{aligned}
$$

for $k=1, \ldots, m-1$, which become the equations in (2.3) upon multiplying by $(\sin \theta)^{-k}$, and

$$
\left(a_{m}(\cos \theta) \cos \frac{\alpha \pi}{2}-b_{m}(\cos \theta) \sin \frac{\alpha \pi}{2}\right)(\sin \theta)^{m}=0,
$$

which gives (2.4). Note that $\sin \theta \neq 0$ as $\theta \in(0, \pi)$.

To proceed from here, we want to choose $2 m$ distinct $\theta_{i}$ such that whenever the polynomials in (2.3) and (2.4) vanish on these $2 m$ points, they will be identically zero. To this end, however, we need to impose an additional symmetry. We choose $\theta_{i}$ to satisfy

$$
\theta_{2 m+1-i}=\pi-\theta_{i}, \quad \theta_{i} \in(0, \pi), \quad 1 \leq i \leq m .
$$

In other words, we choose the latitudes to be symmetric with respect to the equator.

If $p(t)$ is a polynomial of degree $n$, we denote by $p^{\text {even }}$ and $p^{\text {odd }}$ the even and the odd part of $p$, respectively. To be precise, if $p(t)=\sum_{j=0}^{N} a_{j} t^{j}$, then

$$
p^{\text {even }}(t)=\sum_{0 \leq 2 j \leq N} a_{2 j} t^{2 j} \text { and } p^{\text {odd }}(t)=\sum_{1 \leq 2 j-1 \leq N} a_{2 j-1} t^{2 j-1} .
$$

Lemma 2.3. Let $\widetilde{T}_{2 m-1}$ be given as in (2.2). If for some $\theta$ in $(0, \pi)$

$$
\widetilde{T}_{2 m-1}(\theta, \phi)=0, \quad \phi \in \Theta_{0, m} \text { and } \widetilde{T}_{2 m-1}(\pi-\theta, \phi)=0, \quad \phi \in \Theta_{1, m},
$$

then, setting $t=\cos \theta$, we have $a_{0}(t)=0$ and, for $1 \leq k \leq m-1$,

$$
\begin{aligned}
& p_{2 m-k-1}^{\text {even }}(t)+q_{k-1}^{\text {odd }}(t)\left(1-t^{2}\right)^{m-k}=0, \\
& p_{2 m-1-k}^{\text {odd }}(t)+q_{k-1}^{\text {even }}(t)\left(1-t^{2}\right)^{m-k}=0,
\end{aligned}
$$

where either $p_{2 m-k-1}(t)=a_{k}(t)$ and $q_{k-1}(t)=a_{2 m-k}(t)$, or $p_{2 m-k-1}(t)=b_{k}(t)$ and $q_{k-1}(t)=-b_{2 m-k}(t)$. Furthermore, $a_{m}(t)=b_{m}(t)=0$. 
Proof. The assumption allows us to use the previous lemma. Since $\alpha=0$ or $\alpha=1$, the fact that $a_{m}(t)=b_{m}(t)=0$ follows immediately from (2.4). For $1 \leq k \leq m-1$ and $\alpha=0$, the equations (2.3) become

$$
\begin{aligned}
& a_{k}(\cos \theta)+(\sin \theta)^{2 m-2 k} a_{2 m-k}(\cos \theta)=0, \\
& b_{k}(\cos \theta)-(\sin \theta)^{2 m-2 k} b_{2 m-k}(\cos \theta)=0 .
\end{aligned}
$$

For $1 \leq k \leq m-1, \alpha=1$ and $\theta$ replaced by $\pi-\theta$, the equations (2.3) take the form

$$
\begin{aligned}
& a_{k}(-\cos \theta)-(\sin \theta)^{2 m-2 k} a_{2 m-k}(-\cos \theta)=0, \\
& b_{k}(-\cos \theta)+(\sin \theta)^{2 m-2 k} b_{2 m-k}(-\cos \theta)=0 .
\end{aligned}
$$

Since $p(t)+p(-t)=2 p^{\text {even }}(t)$ and $p(t)-p(-t)=2 p^{\text {odd }}(t)$, combining equations (2.7) and (2.8) proves the result.

We use the notation $p_{2 m-k-1}$ and $q_{k-1}$ since they are polynomials of degree $2 m-k-1$ and $k-1$, respectively. In the following we will work with the equations in (2.6). If $p$ is an even polynomial, it can be written as $p(t)=p^{*}\left(t^{2}\right)$; if $p$ is an odd polynomial, it can be written as $p(t)=t p^{*}\left(t^{2}\right)$. Thus, in place of 2.6) we will need to consider polynomials of the form $p(t)+t q(t)\left(1-t^{2}\right)^{r}$ and $t p(t)+q(t)\left(1-t^{2}\right)^{r}$. We will need to study the possibility of interpolation by such polynomials. This is discussed in the following subsection.

2.2. Chebyshev systems. A family of functions $\left\{\phi_{1}, \ldots, \phi_{r}\right\}$ is called a Chebyshev system on a set $E \subseteq \mathbb{R}$, if every linear combination from the $\operatorname{span}\left\{\phi_{1}, \ldots, \phi_{r}\right\}$ has at most $r$ zeros in $E$; in other words, interpolation on $r$ points by functions in the $\operatorname{span}\left\{\phi_{1}, \ldots, \phi_{r}\right\}$ has a unique solution. In this subsection we prove that the families of functions in (2.6) are Chebyshev systems on $(0,1)$.

Proposition 2.4. Let $r$ and $s$ be two nonnegative integers such that $r>s>0$. For $\epsilon=0$ or 1 , let

$$
g(t)=p_{r}\left(t^{2}\right)+t^{ \pm 1}\left(1-t^{2}\right)^{r-s} q_{s-1+\epsilon}\left(t^{2}\right),
$$

where $p_{r}$ and $q_{s-1+\epsilon}$ are polynomials of degree $r$ and $s-1+\epsilon$, respectively. If $g$ vanishes on $r+s+1+\epsilon$ distinct points in $(0,1)$, then $g(t) \equiv 0$.

Proof. We first prove the case that the power of $t^{ \pm 1}$ in $g(t)$ is taken as $t$ and $\epsilon=0$. The cases $t^{-1}$ or $\epsilon=1$ are similar; in fact, the proof for the case $\epsilon=1$ is identical, and only minor changes (merely the numbers $b_{k}^{*}$ below will change) are needed for the case that $t^{ \pm 1}$ is taken as $t^{-1}$.

Changing variables $t \mapsto t^{2}$ shows that we need to prove that if

$$
g^{*}(t)=p_{r}(t)+\sqrt{t}(1-t)^{r-s} q_{s-1}(t)
$$

vanishes on $r+s+1$ distinct points in $[0,1]$, then $g^{*}(t) \equiv 0$. Let

$$
h(t):=t^{r-\frac{1}{2}} \frac{d^{r+1}}{d t^{r+1}} g^{*}(t)=t^{r-\frac{1}{2}} \frac{d^{r+1}}{d t^{r+1}}\left[\sqrt{t}(1-t)^{r-s} q_{s-1}(t)\right] .
$$

Using Rolle's theorem repeatedly, we see that it suffices to prove that if $h(t)$ vanishes on $s$ distinct points in $(0,1)$, then $q_{s-1}(t) \equiv 0$.

Since $q_{s-1}$ is a polynomial, we can write it as

$$
q_{s-1}(t)=b_{0}+b_{1}(1-t)+\ldots+b_{s-1}(1-t)^{s-1} .
$$


Using the Leibnitz rule repeatedly, we have

$$
\frac{d^{r+1}}{d t^{r+1}} t^{k+j+\frac{1}{2}}=\frac{t^{-r-\frac{1}{2}}}{2^{r+1}} \prod_{i=0}^{k+j}(2 i+1)(-1)^{r-k-j} \prod_{i=1}^{r-k-j}(2 i-1) t^{k+j} .
$$

In the following, we will use the convention that $\prod_{i=a}^{b}=1$ whenever $b<a$. This leads to

$$
h(t)=\frac{d^{r+1}}{d t^{r+1}} \sum_{k=0}^{s-1} b_{k} \sum_{j=0}^{r-s}(-1)^{j}\left(\begin{array}{c}
r-j \\
j
\end{array}\right) t^{k+j+\frac{1}{2}}=\sum_{k=0}^{s-1} b_{k}^{*} h_{k}(x)
$$

where

$$
b_{k}^{*}=b_{k} \frac{(-1)^{r-k}}{2^{r+1}} \prod_{i=0}^{k-1}(2 i+1) \prod_{i=1}^{s-k-1}(2 i-1) \quad \text { and } \quad h_{k}(t)=\sum_{j=0}^{r-s} a_{k, j} t^{j+k},
$$

in which the coefficients $a_{k, j}$ are given by

$$
a_{k, j}=\left(\begin{array}{c}
r-s \\
j
\end{array}\right) \prod_{i=0}^{j}(2 k+2 i+1) \prod_{i=j}^{r-s}(2(r-k-i)-1) .
$$

We note that all coefficients $a_{k, j}$ are positive numbers. The polynomial $h_{k}$ is of degree $r-s+k$. In order to prove the proposition, we need to show that the set $\left\{h_{0}, h_{1}, \ldots, h_{s-1}\right\}$ forms a Chebyshev system on $(0,1)$. In other words, we need to prove that the matrix $\left(h_{j}\left(t_{k}\right)\right)_{j, k=0}^{s-1}$ is invertible for any set of distinct points in $(0,1)$.

Let $\mathbf{t}=\left\{t_{1}, t_{2}, \ldots, t_{s}\right\}$ be a given set of distinct numbers in $(0,1)$. For a given set of nonnegative integers $\lambda=\left\{j_{0}, j_{1}, \ldots, j_{s-1}\right\}$, we introduce the notation

$$
V(\lambda ; \mathbf{t})=\operatorname{det}\left[\begin{array}{cccc}
t_{1}^{j_{0}} & t_{2}^{j_{0}} & \ldots & t_{s}^{j_{0}} \\
t_{1}^{j_{1}} & t_{2}^{j_{1}} & \ldots & t_{s}^{j_{1}} \\
\vdots & \vdots & \ldots & \vdots \\
t_{1}^{j_{s-1}} & t_{2}^{j_{s-1}} & \ldots & t_{s}^{j_{s-1}}
\end{array}\right]
$$

In the case of $\lambda=\{s-1, s-2, \ldots, 0\}$, we denote the determinant by $V_{s}(\mathbf{t})$, which is the Vandermonde determinant

$$
V_{s}(\mathbf{t})=\operatorname{det}\left[t_{k+1}^{j}\right]_{k, j=0}^{s-1}=\prod_{1 \leq i<j \leq s}\left(t_{j}-t_{i}\right) .
$$

For a given set of nonnegative integers $\lambda$, we further introduce the notation

$$
s_{\lambda}(\mathbf{t})=s_{j_{0}, j_{1}, \ldots, j_{s-1}}(\mathbf{t})=\frac{V(\lambda ; \mathbf{t})}{V_{s}(\mathbf{t})} .
$$

Note that $s_{\lambda}$ is a symmetric polynomial in $\mathbf{t}$ and $s_{\lambda}$ is zero if $j_{0}, j_{1}, \ldots, j_{s-1}$ are not pairwise distinct. If $\mu=\left(\mu_{0}, \mu_{1} \ldots, \mu_{s-1}\right)$ is a partition, that is, $\mu_{0} \geq \mu_{1} \geq$ $\ldots \geq \mu_{s-1} \geq 0, \mu_{i} \in \mathbb{N}$, and $j_{i}=\mu_{i}+n-i+1$ for $0 \leq j \leq s-1$, then $s_{\lambda}$ is called a Schur polynomial, cf. 9] for details. It is known that Schur polynomials can be written as a linear combination of monomial symmetric polynomials and the coefficients in the linear combination are all positive (called Kosta numbers). For our purpose, it is enough to note that the Schur polynomials are positive when $t_{l}>0$ for all $1 \leq l \leq s$. In particular, it follows that if $j_{0}<j_{1}<\ldots<j_{s-1}$, then $s_{\lambda}(\mathbf{t})$ is positive when $t_{l}>0$ for all $1 \leq l \leq s$. 
Using the definition of the polynomials $h_{j}$ we can write

$$
\begin{aligned}
& \frac{1}{V_{s}(\mathbf{t})} \operatorname{det}\left(h_{j}\left(t_{k+1}\right)\right)_{j, k=0}^{s-1} \\
& \quad=\sum_{j_{0}=0}^{r-s} \sum_{j_{1}=1}^{r-s+1} \ldots \sum_{j_{s-1}=s-1}^{r-1} a_{0, j_{0}} a_{1, j_{1}-1} \ldots a_{s-1, j_{s-1}-(s-1)} s_{j_{0}, j_{1}, \ldots, j_{s-1}}(\mathbf{t}) .
\end{aligned}
$$

For $\lambda=\left\{j_{0}, j_{1}, \ldots, j_{s-1}\right\}$ we will also denote the coefficient of $s_{\lambda}$ in the above sum by $A_{\lambda}$; that is

$$
A_{\lambda}=a_{0, j_{0}} a_{1, j_{1}-1} \ldots a_{s-1, j_{s-1}-(s-1)} .
$$

Recall that $a_{j, k}$ are all positive numbers. If $\lambda=\left\{j_{0}, j_{1}, \ldots, j_{s-1}\right\}$ is not a partition, then a proper permutation of $j_{0}, j_{1}, \ldots, j_{s-1}$ will be. The determinant changes sign when two rows are exchanged, so that $s_{\lambda}$ is positive if the permutation is even and it is negative if the permutation is odd. Every permutation can be factored into a number of transpositions. A transposition means exchanging two elements. If $s_{\lambda}$ is negative, there is a transposition of $\lambda$, call it $\lambda^{\prime}$, such that $s_{\lambda^{\prime}}(\mathbf{t})=-s_{\lambda}(\mathbf{t})>0$.

Let $s_{\lambda}(\mathbf{t})$ be negative and assume that $j_{p}$ and $j_{q}$ are a pair in $\lambda=\left\{j_{0}, j_{1}, \ldots, j_{s-1}\right\}$ such that $p<q$ but $j_{p}>j_{q}$. Considering the summation indices in (2.10), we must have $j_{p}>j_{q}>q>p$. Let $\lambda^{\prime}$ be the image of $\lambda$ under the transposition $(p, q)$, that is, with $j_{p}$ and $j_{q}$ exchanged. Then $s_{\lambda^{\prime}}(\mathbf{t})>0$. The coefficients of these two terms in (2.10), $A_{\lambda}$ and $A_{\lambda^{\prime}}$ differ by two terms only. We have

$$
A_{\lambda^{\prime}}-A_{\lambda}=\left(a_{p, j_{q}-p} a_{q, j_{p}-q}-a_{p, j_{p}-p} a_{q, j_{q}-q}\right) \prod_{i \neq p, q} a_{i, j_{i}-i} .
$$

We now show that $A_{\lambda^{\prime}}>A_{\lambda}$, which will complete the proof of the proposition. Recall the definition of $a_{k, j}$ in (2.9). Let us denote $b_{k, j}=a_{k, j} /\left(\begin{array}{c}r-s \\ j\end{array}\right)$. Then it is easy to verify that

$$
\frac{b_{p, j_{q}-p}}{b_{q, j_{q}-q}}=\frac{(2 q-1)(2 q-3) \ldots(2 p+1)}{(2 s-2 q-1)(2 s-2 q-3) \ldots(2 s-2 p+1)}=: B_{p, q},
$$

which is independent of $j_{p}$ and $j_{q}$. Consequently, we have

$$
\begin{aligned}
\frac{a_{p, j_{q}-p}}{a_{q, j_{q}-q}} & =B_{p, q} \frac{\left(\begin{array}{c}
r-s \\
j_{q}-p
\end{array}\right)}{\left(\begin{array}{c}
r-s \\
j_{q}-q
\end{array}\right)}=B_{p, q}\left(\frac{r-s+1}{j_{q}-q+1}-1\right) \ldots\left(\frac{r-s+1}{j_{q}-p}-1\right) \\
& >B_{p, q}\left(\frac{r-s+1}{j_{p}-q+1}-1\right) \ldots\left(\frac{r-s+1}{j_{p}-p}-1\right)=\frac{a_{p, j_{p}-p}}{a_{q, j_{p}-q}},
\end{aligned}
$$

which implies that $A_{\lambda^{\prime}}>A_{\lambda}$ and completes the proof.

Proposition 2.5. Let $m$ and $k$ be integers such that $1 \leq k \leq m$. Let $p_{2 m-k-1}$ and $q_{k-1}$ be polynomials of degree $2 m-k-1$ and degree $k-1$, respectively. If $t_{1}, \ldots, t_{m}$ be distinct numbers in $(0,1)$ and

$$
\begin{aligned}
& p_{2 m-k-1}^{\text {even }}\left(t_{i}\right)+q_{k-1}^{\text {odd }}\left(t_{i}\right)\left(1-t_{i}^{2}\right)^{m-k}=0, \\
& p_{2 m-1-k}^{\text {odd }}\left(t_{i}\right)+q_{k-1}^{\text {even }}\left(t_{i}\right)\left(1-t_{i}^{2}\right)^{m-k}=0,
\end{aligned}
$$

then $p_{2 m-k-1}(t) \equiv 0$ and $q_{k-1}(t) \equiv 0$.

Proof. Depending on $k$ being even or odd, we need to consider the following four cases. 
CAsE 1. $k$ is even. Setting $r=m-(k+2) / 2$ and $s=(k-2) / 2$, equation (2.11) becomes

$$
p_{r}\left(t_{i}^{2}\right)+t_{i} q_{s}\left(t_{i}^{2}\right)\left(1-t_{i}^{2}\right)^{r-s}=0, \quad 1 \leq i \leq r+s+2 .
$$

From Proposition 2.4 with $\epsilon=1$ and $t^{ \pm 1}=t$, it follows that $p_{r}(t) \equiv 0$ and $q_{s}(t) \equiv 0$.

CASE 2. $k$ is even. Setting $r=m-(k+2) / 2$ and $s=(k-2) / 2$, equation (2.12) becomes

$$
t_{i} p_{r}^{*}\left(t_{i}^{2}\right)+q_{s}^{*}\left(t_{i}^{2}\right)\left(1-t_{i}^{2}\right)^{r-s}=0, \quad 1 \leq i \leq r+s+2 .
$$

Multiplying the equation by $t_{i}^{-1}$, we can use Proposition 2.4 with $\epsilon=1$ and $t^{ \pm 1}=$ $t^{-1}$ to conclude that $p_{r}(t) \equiv 0$ and $q_{s}(t) \equiv 0$.

CASE 3. $k$ is odd. Setting $r=m-(k+1) / 2$ and $s=(k-1) / 2$, equation (2.11) becomes

$$
p_{r}^{*}\left(t_{i}^{2}\right)+t_{i} q_{s-1}^{*}\left(t_{i}^{2}\right)\left(1-t_{i}^{2}\right)^{r-s}=0, \quad 1 \leq i \leq r+s+1 .
$$

From Proposition 2.4 with $\epsilon=0$ and $t^{ \pm 1}=t$, it follows that $p_{r}(t) \equiv 0$ and $q_{s}(t) \equiv 0$. CASE 4. $k$ is odd. Setting $r=m-(k+2) / 2$ and $s=(k-2) / 2$, equation (2.12) becomes

$$
t_{i} p_{r}^{*}\left(t_{i}^{2}\right)+q_{s-1}^{*}\left(t_{i}^{2}\right)\left(1-t_{i}^{2}\right)^{r-s}=0, \quad 1 \leq i \leq r+s+1 .
$$

Multiplying the equation by $t_{i}^{-1}$, we can use Proposition 2.4 with $\epsilon=1$ and $t^{ \pm 1}=t$ to conclude that $p_{r}(t) \equiv 0$ and $q_{s}(t) \equiv 0$.

2.3. Factorization method. The following factorization theorem holds the key to our main result.

Theorem 2.6. Let $m$ and $s$ be positive integers satisfying $m \leq s \leq 2 m-1$. Denote $\lambda=s-m+1$. Let $\theta_{1}, \ldots, \theta_{2 \lambda}$ be distinct numbers in $(0, \pi)$ such that $\theta_{2 \lambda+1-i}=\pi-\theta_{i}$ for $i=1, \ldots, \lambda$. Denote

$$
\widetilde{X}=\left\{\left(\theta_{i}, \phi_{i, j}\right): \phi_{i, j} \in \Theta_{0, m}, 1 \leq i \leq \lambda, \text { and } \phi_{i, j} \in \Theta_{1, m}, \lambda+1 \leq i \leq 2 \lambda\right\} .
$$

If $T_{s} \in \Pi_{s}\left(\mathbb{S}^{2}\right)$ satisfies

$$
\widetilde{T}_{s}\left(\theta_{i}, \phi_{i, j}\right)=0, \quad\left(\theta_{i}, \phi_{i, j}\right) \in \tilde{X},
$$

then there is a spherical polynomial $T_{s-2 \lambda}^{*} \in \Pi_{s-2 \lambda}\left(\mathbb{S}^{2}\right)$ such that

$$
T(x, y, z)=\prod_{i=1}^{2 \lambda}\left(z-\cos \theta_{i}\right), T_{s-2 \lambda}^{*}(x, y, z) .
$$

In particular, $T_{s-2 \lambda}^{*} \equiv 0$ if $s=2 m-1$.

Proof. We start with the formula (2.1), which becomes

$$
\widetilde{T}_{n}(\theta, \phi)=a_{0}(\cos \theta)+\sum_{k=1}^{s}\left[a_{k}(\cos \theta)(\sin \theta)^{k} \cos k \phi+b_{k}(\cos \theta)(\sin \theta)^{k} \sin k \phi\right],
$$

where $a_{k}(\cdot)$ and $b_{k}(\cdot)$ are polynomials of degree $s-k$. For $i=1,2 \ldots, \lambda$, we can follow the proof of Lemma 2.2 and Lemma 2.3 and distinguish the following three cases.

CASE 1. For $0 \leq k \leq 2 m-s-1$,

$$
a_{k}\left(\cos \theta_{i}\right)\left(\sin \theta_{i}\right)^{k}=0, \quad b_{k}\left(\cos \theta_{i}\right)\left(\sin \theta_{i}\right)^{k}=0, \quad i=1, \ldots, 2 \lambda,
$$


setting $b_{0} \equiv 0$. Since $\theta_{i} \in(0, \pi)$, we have that $a_{k}\left(\cos \theta_{i}\right)=0$ and $b_{k}\left(\cos \theta_{i}\right)=0$, for $i=1, \ldots, 2 \lambda$. Recall that $a_{k}$ and $b_{k}$ are polynomials of degree $s-k \geq s-(2 m-$ $s-1)=2 \lambda-1$. Consequently, there exist polynomials $a_{k}^{*}$ and $b_{k}^{*}$, both of degree $s-k-2 \lambda$, such that

$$
a_{k}(t)=\prod_{i=1}^{2 \lambda}\left(t-\cos \theta_{i}\right) a_{k}^{*}(t) \quad \text { and } \quad b_{k}(t)=\prod_{i=1}^{2 \lambda}\left(t-\cos \theta_{i}\right) b_{k}^{*}(t) .
$$

In the extreme case $k=2 m-s-1$, we have $a_{2 m-s-1}=b_{2 m-s-1}=0$.

CASE 2. For $k=m$,

$$
\begin{aligned}
& \left(\sin \theta_{i}\right)^{m} a_{m}\left(\cos \theta_{i}\right)=0 \quad \text { and } \quad i=1, \ldots, \lambda, \\
& \left(\sin \theta_{i}\right)^{m} b_{m}\left(\cos \theta_{i}\right)=0 \quad \text { and } \quad i=\lambda+1, \ldots, 2 \lambda .
\end{aligned}
$$

Since $\theta_{i} \in(0, \pi)$ and both $a_{m}$ and $b_{m}$ are polynomials of degree $s-m=\lambda-1$ vanishing at at least $\lambda$ points, they have to be identically zero.

CASE 3. For $2 m-s \leq k \leq m-1$, we end up with equations similar to (2.6):

$$
\begin{aligned}
& p_{s-k}^{\text {even }}\left(t_{i}\right)+q_{2 \lambda-(s-k)-2}^{\text {odd }}\left(t_{i}\right)\left(1-t^{2}\right)^{s-\lambda-k+1}=0 \\
& p_{s-k}^{\text {odd }}\left(t_{i}\right)+q_{2 \lambda-(s-k)-2}^{\text {even }}\left(t_{i}\right)\left(1-t^{2}\right)^{s-\lambda-k+1}=0,
\end{aligned}
$$

for $i=1,2, \ldots, \lambda$, where either $p_{s-k}(t)=a_{k}(t)$ and $q_{2 \lambda-(s-k)-2}(t)=a_{2 m-k}(t)$, or $p_{s-k}(t)=b_{k}(t)$ and $q_{2 \lambda-(s-k)-2}(t)=-b_{2 m-k}(t)$. In deriving the above equations we have used several times the identity $\lambda=s-m+1$. Recall that $a_{k}$ and $b_{k}$ are polynomials of degree $s-k$; the subscript of the polynomials $p_{2 \lambda-k}$ and $q_{2 \lambda-(s-k)-2}$ again indicate their degree.

It is easy to see that the system of equations (2.13) is exactly the one being studied in the previous subsection, namely (2.11) and (2.12). Hence, using Proposition 2.5 we conclude that

$$
\begin{aligned}
& a_{k}(t) \equiv 0 \quad \text { and } \quad a_{2 m-k}(t) \equiv 0, \quad k=2 m-s, \ldots, m-1 \\
& b_{k}(t) \equiv 0 \quad \text { and } \quad b_{2 m-k}(t) \equiv 0, \quad k=2 m-s, \ldots, m-1 .
\end{aligned}
$$

Together, these three cases show that we have the factorization

$$
\begin{aligned}
\widetilde{T}_{s}(\theta, \phi) & =\prod_{i=1}^{2 \lambda}\left(\cos \theta-\cos \theta_{i}\right) \\
& \times\left(a_{0}^{*}(\cos \theta)+\sum_{k=1}^{2 m-s-2}\left(a_{k}^{*}(\cos \theta) \cos k \phi+b_{k}^{*}(\cos \theta) \sin k \phi\right)\right),
\end{aligned}
$$

which completes the proof.

Using factorization repeatedly, we can obtain a complete factorization of a polynomial of degree $2 m-1$ in $\Pi_{2 m-1}\left(\mathbb{S}^{2}\right)$.

Theorem 2.7. Let $n$ be an odd positive integer, $\sigma \in \mathbb{N}$, and $\lambda_{1}, \ldots, \lambda_{\sigma}$ be positive integers. Define $n_{k}=n_{k-1}-2 \lambda_{k}$, for $1 \leq k \leq \sigma$, with $n_{0}=n$. Assume that $n_{k} \geq 0$ for $1 \leq k \leq \sigma-1$. If $T_{n} \in \Pi_{n}\left(\mathbb{S}^{2}\right)$ satisfies

$$
\widetilde{T}_{n}\left(\theta_{i, k}, \phi_{i, j, k}\right)=0, \quad 1 \leq i \leq 2 \lambda_{k}, 0 \leq j \leq 2\left(n_{k-1}-\lambda_{k}+1\right)-1,1 \leq k \leq \sigma,
$$


where $\theta_{i, k}$ are pairwise distinct angles in $(0, \pi)$ with $\theta_{i, 2 \lambda_{k}+1-l}=\pi-\theta_{i, l}, l=$ $1, \ldots, \lambda_{k}, \phi_{i, j, k} \in \Theta_{0, n_{k-1}-\lambda_{k}+1}$ for $1 \leq i \leq \lambda_{k}$ and $\phi_{i, j, k} \in \Theta_{1, n_{k-1}-\lambda_{k}+1}$ for $\lambda_{k}+1 \leq i \leq 2 \lambda_{k}$, then there exists a polynomial $T_{n_{\sigma}}^{*} \in \Pi_{n_{\sigma}}\left(\mathbb{S}^{2}\right)$ such that

$$
T_{n}(x, y, z)=\prod_{k=1}^{\sigma} \prod_{i=1}^{2 \lambda_{k}}\left(z-\cos \theta_{i, k}\right) T_{n_{\sigma}}^{*}(x, y, z)
$$

In particular, $T_{n}(x, y, z) \equiv 0$ if $n_{\sigma}<0$.

Proof. We apply the factorization result in Theorem 2.6 repeatedly with $s=n_{k-1}$, $m=n_{k-1}-\lambda_{k}+1$ and $\lambda=\lambda_{k}$ for $k=1,2, \ldots, \sigma$.

Just as in the case of an odd number of points on each latitude (cf. 14]), the interpolation nodes in the above theorem are located on latitudes split up into $\sigma$ groups $\left\{\mathbb{S}^{2}\left(z_{i, k}\right): 1 \leq i \leq 2 \lambda_{k}\right\}, 1 \leq k \leq \sigma, z_{i, k}=\cos \theta_{i, k}$, and $z_{2 \lambda_{k}+1-i, k}=-z_{i, k}$. Latitudes in different groups contain a different number of nodes. More precisely, each of the latitudes in the $k$-th group, $\mathbb{S}^{2}\left(z_{1, k}\right), \mathbb{S}^{2}\left(z_{2, k}\right), \ldots, \mathbb{S}^{2}\left(z_{2 \lambda_{k}, k}\right)$, contains an even number of $2\left(n_{k-1}-\lambda_{k}+1\right)$ equidistant points and the points lie on symmetric latitudes. In other words, points on $\mathbb{S}^{2}\left(z_{2 \lambda_{k}+1-i, k}\right)$ and $\mathbb{S}^{2}\left(z_{i, k}\right), i=1, \ldots, \lambda_{k}$, differ by a rotation of an angle of $\pi /\left(2\left(n_{k-1}-\lambda_{k}+1\right)\right)$.

\section{INTERPOLATION ON THE SPHERE}

Our main result on interpolation follows from the factorization Theorem 2.7 The following formula can be used to verify that the number of interpolation conditions matches the dimension of the polynomial space:

$$
\Pi_{s}\left(\mathbb{S}^{2}\right)=\operatorname{dim} \Pi_{s-2 \lambda}\left(\mathbb{S}^{2}\right)+2 \lambda(2 s-2 \lambda+2) .
$$

Theorem 3.1. Let $n$ be an odd natural number and let $\lambda_{1}, \ldots, \lambda_{\sigma}(\sigma \in \mathbb{N})$ be positive integers, such that

$$
\lambda_{1}+\ldots+\lambda_{\sigma}=\frac{n+1}{2} .
$$

Define $n_{k}=n_{k-1}-2 \lambda_{k}$, for $1 \leq k \leq \sigma-1$, with $n_{0}=n$. Let

$$
\widetilde{X}=\left\{\left(\theta_{i, k}, \phi_{i, j, k}\right): 1 \leq i \leq 2 \lambda_{k}, 0 \leq j \leq 2\left(n_{k-1}-\lambda_{k}+1\right)-1,1 \leq k \leq \sigma\right\},
$$

where $\theta_{i, k}, 1 \leq j \leq 2 \lambda_{k}$ and $1 \leq k \leq \sigma$, are distinct numbers in $(0, \pi)$ with $\theta_{2 \lambda_{k}+1-i, k}=\pi-\theta_{i, k}\left(i=1, \ldots, \lambda_{k}\right), \phi_{i, j, k} \in \Theta_{0, n_{k-1}-\lambda_{k}+1}$, for $1 \leq i \leq \lambda_{k}$, and $\phi_{i, j, k} \in \Theta_{1, n_{k-1}-\lambda_{k}+1}$, for $\lambda_{k}+1 \leq i \leq 2 \lambda_{k}$. Then the set $X$ solves the interpolation problem in $\Pi_{n}\left(\mathbb{S}^{2}\right)$.

Proof. First, we verify that the dimension of $\Pi_{n}\left(\mathbb{S}^{2}\right)$ matches the number of interpolation conditions. Let $|X|$ denote the number of points in $X$. It follows from equation (3.1) that

$$
\begin{aligned}
|X| & =\sum_{k=1}^{\sigma} 2 \lambda_{k}\left(2 n_{k-1}-2 \lambda_{k}+2\right) \\
& =\sum_{k=1}^{\sigma}\left(\operatorname{dim} \Pi_{n_{k-1}}\left(\mathbb{S}^{2}\right)-\operatorname{dim} \Pi_{n_{k-1}-2 \lambda_{k}}\left(\mathbb{S}^{2}\right)\right)=(n+1)^{2}=\operatorname{dim} \Pi_{n}\left(\mathbb{S}^{2}\right) .
\end{aligned}
$$


Thus, it is sufficient to show that if $T_{n} \in \Pi_{n}\left(\mathbb{S}^{2}\right)$ vanishes on $X$, then $T_{n}(x, y, z) \equiv 0$. Under the condition (3.2), it follows that

$$
n_{\sigma}=n_{\sigma-1}-2 \lambda_{\sigma}=n_{\sigma-2}-2 \lambda_{\sigma-1}-2 \lambda_{\sigma}=\cdots=n-2 \sum_{k=1}^{\sigma-1} \lambda_{k}=-1<0 .
$$

Hence, the factorization in Theorem 2.7 shows that $T(x, y, z) \equiv 0$.

For a fixed $n=2 m-1$, this theorem contains a number of different interpolation processes. In fact, for each positive odd integer $n$, the number of point sets $X$ which can be deduced from Theorem 3.1 depends on the partition number of $(n+$ 1)/2. Every solution of equation (3.2) leads to a set of points defining a poised interpolation problem in $\Pi_{n}\left(\mathbb{S}^{2}\right)$. The number of solutions of such an equation grows exponentially as $n$ goes to infinity. Moreover, the order of $\lambda_{1}, \ldots, \lambda_{\sigma}$ matters; i.e. , different permutations of a solution $\lambda_{1}, \ldots, \lambda_{\sigma}$ of equation (3.2) give different sets of interpolation points.

Among the solutions of equation (3.2), one extreme case is $\sigma=1$, for which the equation has only one solution $\lambda_{1}=(n+1) / 2$. In this case, the interpolation points are located on $n+1$ symmetric latitudes $\mathbb{S}^{2}\left(z_{1}\right), \mathbb{S}^{2}\left(z_{2}\right), \ldots, \mathbb{S}^{2}\left(z_{n+1}\right)$, each of them containing $n+1$ equidistant points. This case has already appeared in 4, Theorem $2.5]$.

The other extreme case is $\sigma=(n+1) / 2$ and $\lambda_{1}=\cdots=\lambda_{(n+1) / 2}=1$. There, $n_{k}=n-2 k$ and we have $(n+1) / 2$ groups of two symmetric latitudes, where the ones in the $k$ th group contain $2(n-2 k+2)$ equidistant nodes. The points on a latitude are rotated by an angle of $\pi /(2(n-2 k+2))$ with respect to the points on the corresponding symmetric latitude of the same group.

Example 3.2. To illustrate the power of the factorization method, we present the possible point distributions for $n=3,5$ and 7 .

- $n=3$

(1) $\sigma=1, \lambda_{1}=2: 4$ latitudes each with 4 points,

(2) $\sigma=2, \lambda_{1}=\lambda_{2}=1: 2$ latitudes with 6 points and 2 latitudes with 2 points.

- $n=5$

(1) $\sigma=1, \lambda_{1}=3: 6$ latitudes, each with 6 points.

(2) $\sigma=2, \lambda_{1}+\lambda_{2}=3$ has two solutions.

(a) $\lambda_{1}=1, \lambda_{2}=2: 4$ latitudes with 8 points and 2 latitudes with 2 points.

(b) $\lambda_{1}=2, \lambda_{2}=1: 2$ latitudes with 10 points and 4 latitudes with 4 points.

(3) $\sigma=3, \lambda_{1}=\lambda_{2}=\lambda_{3}=1$ : 2 latitudes with 10 points, 2 latitudes with 6 points and 2 latitudes with 2 points.

- $n=7$

(1) $\sigma=1, \lambda_{1}=4: 8$ latitudes with 8 points.

(2) $\sigma=2, \lambda_{1}+\lambda_{2}=4$ has three solutions,

(a) $\lambda_{1}=2, \lambda_{2}=2: 4$ latitudes with 12 points and 4 latitudes with 4 points;

(b) $\lambda_{1}=1, \lambda_{2}=3: 2$ latitudes with 14 points and 6 latitudes with 6 points; 
(c) $\lambda_{1}=3, \lambda_{2}=1: 6$ latitudes with 10 points and 2 latitudes with 2 points.

(3) $\sigma=3, \lambda_{1}+\lambda_{2}+\lambda_{3}=4$ has three solutions,

(a) $\lambda_{1}=1, \lambda_{2}=1, \lambda_{3}=2$ : 2 latitudes with 14 points, 2 latitudes with 10 points and 4 latitudes with 4 points.

(b) $\lambda_{1}=1, \lambda_{2}=2, \lambda_{3}=1$ : 2 latitudes with 14 points, 4 latitudes with 8 points and 2 latitudes with 2 points;

(c) $\lambda_{1}=2, \lambda_{2}=1, \lambda_{3}=1: 4$ latitudes with 12 points, 2 latitudes with 6 points and 2 latitudes with 2 points.

(4) $\sigma=4, \lambda_{1}=\lambda_{2}=\lambda_{3}=\lambda_{4}=1: 2$ latitudes with 14 points, 2 latitudes with 10 points, 2 latitudes with 6 points and 2 latitudes with 4 points.

It is well-known that interpolating polynomials can be used to construct cubature formulas on the unit sphere (cf. [12]). In fact, integrating the interpolation polynomial in $\Pi_{n}\left(\mathbb{S}^{2}\right)$ yields a cubature formula on the sphere which is exact for spherical polynomials of degree $n$. Among the point sets in Theorem 3.1 the case where the points are distributed on $2 m$ symmetric latitudes, with each latitude containing $2 m$ equidistant points, is of particular interest. In this case, the cubature formula is simple and can be explicitly given.

Proposition 3.3. Let $m$ be a positive integer. Let $\theta_{1}, \ldots, \theta_{2 m}$ be pairwise distinct numbers in $(0, \pi)$ with $\theta_{2 m+1-i}=\pi-\theta_{i}, i=1, \ldots, m$, and $\alpha \in\{0,1\}$. Then for all $T_{2 m-1} \in \Pi_{2 m-1}\left(\mathbb{S}^{2}\right)$,

$$
\int_{\mathbb{S}^{2}} T_{2 m-1}(\xi) d \omega(\xi)=\frac{\pi}{m} \sum_{i=1}^{m} \lambda_{i} \sum_{j=0}^{2 m-1} \widetilde{T}_{2 m-1}\left(\theta_{i}, \phi_{j}^{0}\right)+\frac{\pi}{m} \sum_{i=m+1}^{2 m} \lambda_{i} \sum_{j=0}^{2 m-1} \widetilde{T}_{2 m-1}\left(\theta_{i}, \phi_{j}^{1}\right)
$$

where $\phi_{j}^{\alpha}=(2 j+\alpha) \pi / 2 m$, and $\lambda_{i}$ is given by

$$
\lambda_{i}=\int_{-1}^{1} \prod_{k=1, k \neq i}^{2 m} \frac{t-\cos \theta_{k}}{\cos \theta_{i}-\cos \theta_{k}} d t, \quad i=1, \ldots, 2 m .
$$

Proof. Let the interpolation polynomial $T_{2 m-1}$ be of the form (2.1). We use the quadrature formula

$$
\frac{1}{2 \pi} \int_{0}^{2 \pi} p(t) d t=\frac{1}{2 m} \sum_{j=0}^{2 m-1} p\left(\phi_{j}^{\alpha}\right)
$$

which is known to hold for every trigonometric polynomial of degree at most $m$ (see, for example, [16, Vol.2, p. 8]). Using formula (3.3) and the interpolation property of $T_{2 m-1}$, it follows that

$$
a_{0}\left(\cos \theta_{i}\right)=\frac{1}{2 \pi} \int_{0}^{2 \pi} \widetilde{T}_{2 m-1}\left(\theta_{i}, \phi\right) d \phi=\frac{1}{2 m} \sum_{j=0}^{2 m-1} \widetilde{T}_{2 m-1}\left(\theta_{i}, \phi_{j}^{\alpha}\right),
$$

for every fixed $\theta_{i}, 1 \leq i \leq 2 m$. Consequently, $a_{0}$, which is a polynomial of degree $2 m-1$ in one variable, is uniquely determined by these $2 m$ interpolation conditions. It follows that

$$
a_{0}(t)=\sum_{i=1}^{m}\left(\frac{1}{2 m} \sum_{j=0}^{2 m-1} \widetilde{T}_{2 m-1}\left(\theta_{i}, \phi_{j}^{0}\right)\right) \ell_{i}(t)+\sum_{i=m+1}^{2 m}\left(\frac{1}{2 m} \sum_{j=0}^{2 m-1} \widetilde{T}_{2 m-1}\left(\theta_{i}, \phi_{j}^{1}\right)\right) \ell_{i}(t),
$$


where $\ell_{i}(t)=\prod_{k=1, k \neq i}^{2 m}\left(t-\cos \theta_{k}\right) /\left(\cos \theta_{i}-\cos \theta_{k}\right)$. Using the change of variables

$$
\int_{\mathbb{S}^{2}} T_{2 m-1}(\xi) d \omega(\xi)=\int_{0}^{\pi} \int_{0}^{2 \pi} \widetilde{T}_{2 m-1}(\theta, \phi) \sin \theta d \phi d \theta
$$

the integral of $T_{2 m-1}$ over the surface of the sphere is equal to

$$
\int_{\mathbb{S}^{2}} T_{2 m-1}(\xi) d \omega(\xi)=2 \pi \int_{0}^{\pi} a_{0}(\cos \theta) \sin \theta d \theta=2 \pi \int_{-1}^{1} a_{0}(t) d t .
$$

The stated formula follows from the formula for $a_{0}(t)$ given above.

In particular, this result shows that the cubature formula is nonnegative, if $\cos \theta_{i}$ are chosen so that $\lambda_{i}$ are nonnegative. This holds, for example, if $\cos \theta_{i}$ are the zeros of the Legendre polynomial $P_{2 m}$ of degree $2 m$, or the zeros of a quasi Legendre orthogonal polynomial $P_{2 m}+\alpha P_{2 m-1}$ with mild conditions imposed on $\alpha \in \mathbb{R}$ (see, for example, [13]). In [10, the positivity of the cubature in this case has been proved by working directly with the interpolation matrix.

\section{REFERENCES}

[1] B. Bojanov and Yuan $\mathrm{Xu}$, On a Hermite interpolation by polynomials of two variables, SIAM J. Numer. Anal. 39 (2002), 1780-1793.

[2] B. Bojanov and Yuan Xu, Polynomial interpolation of two variables based on a factorization method, J. Approx. Theory, 120 (2003), p. 267-282.

[3] N. Laín Fernández, Polynomial bases on the sphere, Proceedings IDoMAT 2001, 142, pages 39-52, Birkhäuser, 2001

[4] N. Laín Fernández Polynomial bases on the sphere, Phd Thesis, Universität Lübeck, 2003

[5] N. Laín Fernández and J. Prestin, Interpolatory band-limited wavelets on the sphere, submitted to Constr. Approx..

[6] M. Gasca and T. Sauer, Polynomial interpolation in several variables, Adv. in Comput. Math., 12 (2000), 377-410.

[7] M. v. Golitschek and W. A. Light, Interpolation by polynomials and radial basis functions on spheres, Constr. Approx. 17 (2001), 1-18.

[8] H. Hakopian and S. Ismaeil, On a bivariate interpolation problem, J. Approx. Theory, 116 (2002), 76-99.

[9] I. G. Macdonald, Symmetric functions and Hall polynomials, 2ed ed. Oxford Mathematical Monographs, Clarendon Press, New York, 1995.

[10] J. Prestin and D. Rosca, On a positive cubature formula on the sphere, preprint.

[11] I. H. Sloan and R. S. Womersley, How good can polynomial interpolation on the sphere be? Adv. Comp. Math. 14 (2001), 195-226.

[12] A. Stroud, Approximate calculation of multiple integrals, Prentice Hall, Englewood Cliffs, NJ, 1971.

[13] Yuan Xu, A Characterization of positive quadrature formulae, Math. Comp. 62 (1994), 703718.

[14] Yuan Xu, Polynomial interpolation on the unit sphere, SIAM J. Numer. Anal. 41 (2003), 751-766.

[15] Yuan Xu, Polynomial interpolation on the unit ball and on the unit sphere, Adv. in Comp. Math., 20 (2004), 247-260.

[16] A. Zygmund, Trigonometric series, Cambridge University Press, Cambridge, 1959.

Institute of Biomathematics and Biometry, GSF - National Research Center for Environment and Health, 85764 Neuherberg, Germany

E-mail address: castell@gaf.de

Center for Mathematical Sciences, Munich University of Technology, Boltzmannstr. 3, 85747 Munich, Germany

E-mail address: fernande@ma.tum.de 
Department of Mathematics, University of Oregon, Eugene, Oregon 97403-1222.

E-mail address: yuan@math.uoregon.edu 